\title{
Vocal Cord Leukoplakia: Characteristics and Pathological Significance
}

\author{
Yusuf Kizil, Utku Aydil, Metin Yilmaz, Özgür Ekinci, Osman Tugrul Güzeldir, Veysel Akif Savas, Ahmet Köybasioglu
}

\begin{abstract}
Leukoplakia is a nonspecific clinical term used to describe a mucosal white patch or plaque that cannot be easily scraped off. Leukoplakia of vocal cord represents a chronic inflammation or exposure to irritants which can also stimulate development of precancerous conditions or cancer. This study aimed to determine clinical and histo pathological characteristics of vocal cord leukoplakia. A total of 66 patients were included. All patients with a clinical diagnosis of vocal cord leukoplakia who had undergone direct laryngoscopic examination and biopsy were analyzed retrospectively. The most common pathological finding was mild dysplasia (25.8\%). Cancer was detected in $18.2 \%$ of cases. Malignancy was more frequent in cases with unilateral vocal cord involvement (23.4\%) when compared with bilateral cases $(5.2 \%)$ and in patients with localized lesions (19.3\%) when compared with lesions involving whole cord (11.1\%), but the differences were not statistically significant $(p>0.05)$. S moking history was found to be related with cancer diagnosis $(p<0.001)$. In the presence of a serious smoking history, there is high-risk for malignancy and leukoplakia should be sampled immediately for histopathological examination. Although statistically not proven, cancer rates are higher in more localized and unilateral lesions.
\end{abstract}

Keywords: Vocal cords, Leukoplakia, Larynx, Laryngeal epithelium, Preneoplastic condition.

How to cite this article: Kizil Y, Aydil U, Yilmaz M, Ekinci Ö, Güzeldir OT, Savas VA, Köybasioglu A. Vocal Cord Leukoplakia: Characteristics and Pathological Significance. Int J Phonosurg Laryngol 2012;2(1):9-13.

\section{Source of support $\mathrm{Nil}$}

\section{Conflict of interest: None}

\section{INTRODUCTION}

Leukoplakia can be described as a mucosal white patch or plaque that cannot be easily scraped off. ${ }^{1}$ It is a clinical term and does not have a special pathological equival ent. ${ }^{1}$ Although the term, leukoplakia, is generally used for oral and laryngeal lesions, it can be used to describe other white patches seen in gastrointestinal and genitourinary tract. Since, it is a nonspecific clinical term, leukoplakia is widely used in otolaryngology without consideration of etiology and histological features. Sometimes, terms of histopathological diagnosis, such as keratosis, hyperkeratosis and dysplasia are used instead of leukoplakia and terminological confusion arises. ${ }^{1}$ In otolaryngological practice, leukoplakia of vocal cords is a remarkable and significant clinical finding. M ore or less, leukoplakia of vocal cord represents a chronic inflammation or exsposure to irritants which can also stimulate development of precancerous conditions or cancer. Smoking and other inhaled irritants, alcohol consumption, laryngopharyngeal reflux and infectious conditions are among common causes.

Histopathological diagnosis of a cancer or a premalignant condition in patients with vocal cord leukoplakia is crucial for prevention or early diagnosis of laryngeal squamous cell carcinoma (SCC). The survival rate of patients with laryngeal SCC has not significantly improved over the last decades despite advances in medical technologies and oncology. ${ }^{2}$ For cancer of the larynx, early recognition is still the key to success. In this study, we aim to describe clinical characteristics and histopathological examination results of vocal cord leukoplakias.

\section{MATERIALS AND METHODS}

\section{Subjects and Inclusion C riteria}

The medical records of the patients who had surgery for vocal cord leukoplakia between J anuary 2000 and M arch 2011 at our institution were retrospectively reviewed after Institutional Ethical Board approval. Our policy for vocal cord leukoplakia sampling is total cold knife excision of the lesion for histopathological examination. Initially, 94 patients were identified, but only 66 of these patients fulfilled the study inclusion criteria. Inclusion criteria were:

a. Persistent leukoplakia of true vocal cords at least for 2 months

b. A bsence of erythroplakia

c. A bsence of an obvious mass lesion or exophytic lesion of vocal cords

d. A bsence of previous head and neck malignancy

e. A bsence of previous endolaryngeal surgery

f. A bsence of history of radiation therapy to head and neck region

g. Normal bilateral vocal fold mobility

h. A bsence of any other synchronous undefined laryngeal lesions.

\section{Review Process}

Demographic features, symptoms, localization and extension of leukoplakia according to laryngoscopic examination, smoking habits and pathological examination results were reviewed. For definition of lesion extention, 
each vocal cord was divided into three equal segments: A nterior, middle and posterior. F requencies of involvement of totally six parts on the two vocal cords were determined for each patient. Involvement of all three segments on the same vocal cord was defined as total involvement. Relation between total involvement of vocal cord(s) by leukoplakia and cancer detection rate was statistically analyzed. Cancer detection rates among cases with unilateral and bilateral involvement of the cords were also compared.

Histopathological examination results were classified according to the most severe form of the lesion for each patient. The histopathological findings were graded from mild to severe as inflammatory changes, hyperkeratosis, squamous hyperplasia, mild dysplasia, moderate dysplasia, severe dysplasia, carcinoma insitu, microinvasive SCC and invasive SCC. These findings were classified in four groups: inflammatory changes, hyperkeratosis, squamous hyperplasia in mild changes group (group 1); mild dysplasia and moderate dysplasia in early precancerosis group (group 2); severe dysplasia and carcinoma in situ in advanced preinvasive lesions group (group 3) and microinvasive SCC and invasive SCC in cancer group (group 4).

Patients were al so grouped according to smoking history. Four groups consisted of patients with more than 40 packyear smoking history, between 20 and 40 pack-year smoking history, less than 20 pack-year smoking history and without a smoking history.

\section{Statistical Analysis}

In statistical analysis, Fisher's exact test was used for categorical variables, and $M$ ann-W hitney test was used for continuous variables since the data was not normally distributed. The $p$-values of $\leq 0.05$ were considered to be statistically significant.

\section{RESULTS}

Totally, 66 patients ( 61 male and 5 female) met the inclusion criteria for analysis. M ean age of the patients was 54.8 years

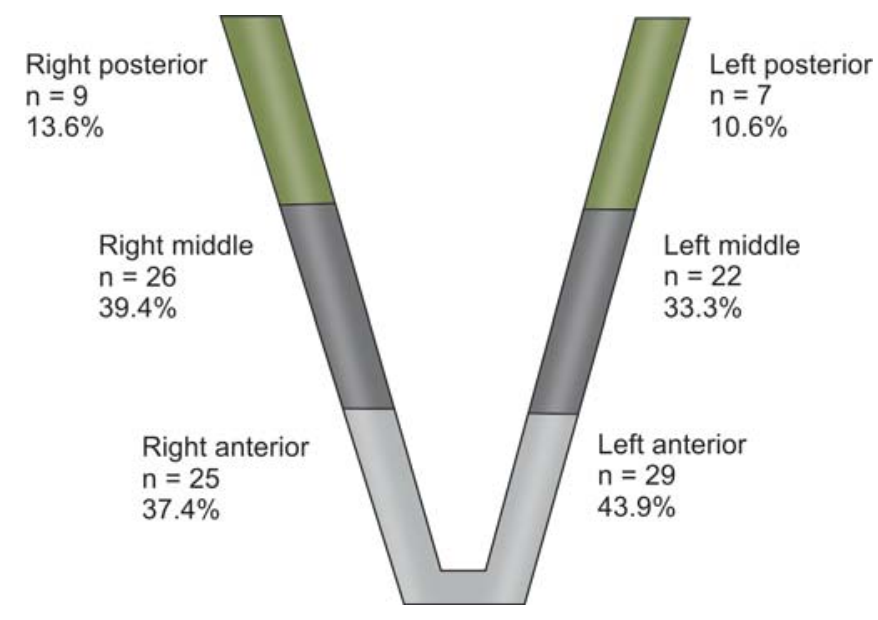

Fig. 1: Localization of leukoplakia on vocal cords

(ranged between 35 and 76 years, SD, 10.1). M ost common presenting symptom was hoarseness in $59(89.4 \%)$ patients and globus sensation was encountered in seven patients (10.6\%). Nineteen patients (28.8\%) had reflux symptoms besides voice change.

Most common site of involvement was left anterior (43.9\%; Fig. 1). Posterior segment involvement was rare and posterior of the right cord and the left cord were involved in 13.6 and $10.6 \%$ of the cases respectively. The most common histopathological finding was mild dysplasia found in 17 patients (25.8\%; Table 1 ). M icroinvasive SCC was reported in four patients $(6.1 \%)$ and invasive SCC in eight patients (12.1\%).

In 19 patients (28.8\%), vocal cords were involved bilaterally. Cancer development was reported in one (5.2\%) of 19 patients with bilateral involvement and 11 (23.4\%) of 47 patients with unilateral involvement. The difference between the two groups was not statistically significant (Fisher's exact test $\chi^{2}=2.99, p=0.156$ ).

Total involvement of vocal cord was detected unilaterally in five patients (7.6\%) and bilaterally in four patients (6.1\%). Cancer (microinvasive or invasive SCC)

Table 1: Histopathological examination results (SCC: Squamous cell carcinoma)

\begin{tabular}{|c|c|c|c|c|c|}
\hline Pathological results & $\mathrm{n}$ & $\%$ & Groups & $\mathrm{n}$ & $\%$ \\
\hline Inflammatory changes & 10 & 15.1 & Group I (mild changes group) & 22 & 33.3 \\
\hline Hyperkeratosis & 4 & 6.1 & & & \\
\hline Squamous hyperplasia & 8 & 12.1 & & & \\
\hline Mild dysplasia & 17 & 25.8 & Group II (early precancerosis group) & 26 & 39.4 \\
\hline Moderate dysplasia & 9 & 13.6 & & & \\
\hline Severe dysplasia & 5 & 7.6 & Group III (advanced preinvasive lesions group) & 6 & 9.1 \\
\hline Carcinoma in situ & 1 & 1.5 & & & \\
\hline Microinvasive SCC & 4 & 6.1 & Group IV (cancer group) & 12 & 18.2 \\
\hline Invasive SCC & 8 & 12.1 & & & \\
\hline Total & 66 & 100.0 & & 66 & 100.0 \\
\hline
\end{tabular}


Vocal Cord Leukoplakia: Characteristics and Pathological Significance

Table 2: Comparison of histopathological examination results with smoking history

\begin{tabular}{llllll}
\hline S moking history & Group I (\%) & Group II (\%) & Group III (\%) & Group IV (\%) & Total \\
\hline Heavy smoker (> 40 pack-year) & $1(7.1)$ & $4(28.6)$ & $1(7.1)$ & $8(57.2)$ & $14(100.0)$ \\
Moderate smoker (20-40 pack-year) & $5(33.3)$ & $6(40.0)$ & $1(6.7)$ & $3(20.0)$ & $15(100.0)$ \\
Light smoker (<20 pack-year) & $9(40.9)$ & $12(54.5)$ & 0 & $1(4.6)$ & $22(100.0)$ \\
No smoking history & $7(46.7)$ & $5(33.3)$ & $3(20.0)$ & 0 & $15(100.0)$ \\
\hline
\end{tabular}

was present in $11(19.3 \%)$ of 57 patients with partial vocal cord involvement and in one (11.1\%) of nine patients with total vocal cord involvement. The difference between the two groups was not statistically significant (Fisher's exact test $\chi^{2}=0.35, p=1.0$ ).

When patients were classified according to smoking history, most common histological diagnosis was either microinvasive SCC or invasive SCC in heavy smokers whereas either group I or group II pathologies were more common in less smokers (Table 2). Difference in cancer frequency between nonsmokers (group IV) and with smoking history (groups I, II, III) was statistically significant ( $M$ ann-W hitney test, $u=74.0, p<0.001$ ).

\section{DISCUSSION}

Leukoplakia can be a clinical representation of a benign pathological situation, such as inflammation, any level in carcinogenic transformation or a cancer. For determining accurate histological diagnosis of leukoplakia, light microscopic examination still remains the most common and reliable method. Histopathological grading of leukoplakia is not uniform but it is mainly related to the potential for malignant transformation. There are more than 20 classifications of laryngeal epithelial lesions worldwide but two of them are more commonly used: WHO dysplasia grading system and Ljubljana classification. ${ }^{1,3}$ In WHO dysplasia classification system, categories are hyperplasia with increased number of cells; mild, moderate and severe dysplasia; and carcinoma in situ. L jubljana classification includes mainly three groups: lesions with minimal risk of progression to invasive carcinoma, including squamous and basal-parabasal cell hyperplasia; potentially malignant group (atypical hyperplasia or risky epithelium); and carcinoma insitu. Whatever, the system used in histological evaluation and classification, severity of changes in laryngeal epithelium can be put into a scale from mildest to most severe according to potential biological behavior. In the present study, we put the histological findings into an order according to malign transformation potential and degree of changes in normal epithelial architecture. Only the most severe change was taken into account. The first three degrees were inflammation, keratosis and epithelial hyperplasia without atypia. These $3^{\circ}$ constituted the mild changes group with no or minimal risk of malignant transformation. We separated severe dysplasia from the mild and the moderate forms and evaluated with carcinoma insitu in advanced preinvasive lesions group. The mild and the moderate degrees of dysplasia were grouped as early precancerosis group. The rationale for this grouping is difficulty of distinguishing between severe dysplasia and carcinoma in situ histopathologically and common coexistence of these two histopathological diagnoses in the same pathological specimen. Cancer group was consisted of microinvasive and invasive forms of SCC.

Recently, noninvasive and convenient diagnostic methods for detection of laryngeal dysplasia and cancer development are evolving. These include touch smear cytology, optical coherence tomography, narrow band imaging, fluorescence laryngoscopy and contact endoscopy. ${ }^{4-9} \mathrm{H}$ owever, noneof theseare in common clinical use so biopsy and histopathological examination are still the mainstay of the diagnostic management process.

When a vocal cord leukoplakia accompanies an irregular mass lesion on the vocal cords, high clinical suspicion for laryngeal cancer arises. In that case management strategy is not so debatable. However, if a vocal cord leukoplakia only appearing like a patch or simple whitening of mucosa, there are different management strategies including followup without any medication and cessation of carcinogens, such as tobacco and alcohol; treatment with retinyl palmitate, proton pump inhibitors; direct laringoscopy and biopsy for histological diagnosis; excisional biopsy; ablation with laser and stripping. ${ }^{10-14} \mathrm{~A}$ mong these, follow-up with cessation of possible carcinogens, such as tobacco and alcohol together with treating irritating acid reflux is a common strategy. However, clinical characteristics of the disease should be well known not to lose time in case of a cancer or a high-risk lesion of cancer development. Head and neck cancers are more frequent among people with low socioeconomic status and it is not infrequent to see non compliant patients who do not attend follow-up visits regularly and do not change smoking and drinking habits. So that, we planned this study to determine clinical characteristics of vocal cord leukoplakia which may harbor a cancer or carry a high-risk for cancer development. 
In English literature, studies regarding vocal cord leukoplakias are scarce. $M$ any of the studies are about vocal cord dysplasias and malignant transformation rates of dysplastic lesions of vocal cords in time. Localization of the vocal cord leukoplakia is also rarely studied to date. Superior surface and anterior parts of vocal cords reported to be more involved by glottic dysplasia but the data regarding common localizations of vocal cord leukoplakia is not present according to our knowledge. ${ }^{15,16}$ In the present study, we have found that, like leukoplakias are commonly located on anterior and medial parts of the cords and posterior involvement was rare. A comprehensive review of laryngeal leukoplakia by I senberg et al was published in 2008. ${ }^{17}$ A ccording to this review, mild/moderate dysplasia and severe dysplasia were found in 33.5 and $15.2 \%$ of the laryngeal leukoplakia biopsy specimens respectively. The overall malignant transformation rate reported was $8.2 \%$ in vocal cord dysplasias. M alignant transformation rates during follow-up were $3.7,10.1$ and $18.1 \%$ in patients with no dysplasia, with mild and moderate dysplasia and with severe dysplasia respectively. In this review, the authors did not report the rate of cancer at initial biopsy. Total frequency of dysplasias and carcinoma in situ in our study is $48.5 \%$ and consistent with the rates reported in this review. In our study, cancer was detected in $18.2 \%$ of the patients with vocal cord leukoplakia and it is relatively high.

In this study, we observed that localized and unilateral lesions are more related with cancer development however, this relation could not be proved statistically. Future studies may show possible relationship between localized lesions with increased cancer development risk. Strong relationship of severe smoking history with high cancer development rate within the vocal cord leukoplakia was documented in our study, so that a high-risk group is well-defined. A lthough the difference was not statistically significant, we recommend biopsy of a localized and unilateral leukoplakia immediately when smoking history is present.

\section{REFERENCES}

1. Gale N, Zidar N. Benign and potentially malignant lesions of the squamous epithelium and squamous cell carcinoma. In: Cardesa A, Slootweg PJ (Eds). Pathology of the Head and Neck. Springer, B erlin-Heidel berg 2006;1-29.

2. Carval ho A L, Nishimoto IN , Califano JA, K owalski L P. Trends in incidence and prognosis for head and neck cancer in the U nited States: A site-specific analysis of the SEER database. Int J Cancer 2005; 114:806-16.

3. Hellquist H, Cardesa A, Gale N, Kambic V , M ichaels L. Criteria for grading in the Ljubljana classification of epithelial hyperplastic laryngeal lesions. A study by members of the
W orking Group on Epithelial Hyperplastic Laryngeal Lesions of the European Society of Pathology. Histopathology 1999;34: 226-33.

4. U stundag E, Kaur A C, B oyaci Z, K eskin G, A ydin O. Combined use of histopathology with touch smear cytology in biopsies of the Iarynx. Eur Arch Otorhinolaryngol 2006;263: 866-71.

5. K raft M, Glanz H, von Gerlach S, Wisweh $H$, L ubatschowski $\mathrm{H}, \mathrm{A}$ rens $\mathrm{C}$. Clinical value of optical coherence tomography in laryngology. Head Neck 2008;30:1628-35.

6. Watanabe $A$, Taniguchi $M$, T sujie $H$, Hosokawa M, Fujita M , Sasaki $S$. The value of narrow band imaging for early detection of laryngeal cancer. Eur A rch Otorhinolaryngol 2009;266: 1017-23.

7. A rens $C$, R eussner $D$, W oenkhaus J L L eunig A, B etz CS, G lanz $\mathrm{H}$. Indirect fluorescence laryngoscopy in the diagnosis of precancerous and cancerous laryngeal lesions. Eur A rch Otorhinolaryngol 2007;264:621-26.

8. W arnecke A, A verbeck T, L einung $M$, Soudah B, W enzel GI, $\mathrm{K}$ reipe $\mathrm{HH}$, Lenarz $\mathrm{T}$, Stöver $\mathrm{T}$. Contact endoscopy for the evaluation of the pharyngeal and laryngeal mucosa. L aryngoscope 2010;120:253-58.

9. Malzahn K, Dreyer T, Glanz H, A rens C. A utofluorescence endoscopy in the diagnosis of early laryngeal cancer and its precursor lesions. Laryngoscope 2002;112:488-93.

10. Issing WJ , Struck R, Naumann A. L ong-term follow-up of larynx leukoplakia under treatment with retinyl palmitate. Head N eck 1996;18:560-65.

11. Simpson CB, A rchilla AS, V elázquez RA, McGuff HS. Resolution of vocal fold leukoplakia with proton-pump inhibitor therapy. Ear Nose Throat] 2006;85:362-64.

12. M inni $A, B$ arbaro $M$, Rispoli $G$, Diaferia $F, B$ ernardeschi $D$, Filipo R. Treatment with laser $\mathrm{CO}_{2}$ cordectomy and clinical implications in management of mild and moderate laryngeal precancerosis. Eur A rch Otorhinolaryngol 2008;265:189-93.

13. Gallo A, de V incentiis M, D ella Rocca C, M oi R, Simonelli M, $M$ inni $A$, Shaha AR. Evolution of precancerous laryngeal lesions: A clinicopathologic study with long-term follow-up on 259 patients. Head Neck 2001;23:42-47.

14. Sadri M, M CM ahon J, Parker A. M anagement of laryngeal dysplasia: A review. Eur Arch Otorhinolaryngol 2006;263: 843-52.

15. L ahav $Y, B$ urns JA, Feinberg S, Heaton JT, Zeitels SM . Initial anatomic geographic presentation of glottal dysplasia. A nn O tol Rhinol Laryngol 2009;118:321-25.

16. Zeitels SM . Premalignant epithelium and microinvasive cancer of the vocal fold: The evolution of phonomicrosurgical management. Laryngoscope 1995;105(S1):1-44.

17. I senberg J S, Crozier DL, Dailey SH. Institutional and comprehensive review of laryngeal leukoplakia. Ann Otol Rhinol Laryngol 2008;117:74-79.

\section{ABOUT THE AUTHORS}

\section{Yusuf Kizil (Corresponding Author)}

Department of O torhinolaryngology, G azi U niversity, T urkey, e-mail: yusufkizil@yahoo.com 


\section{Utku Aydil}

Department of Otorhinolaryngology, G azi University, Turkey

\section{Metin Yilmaz}

Professor, Departmentof Otorhinolaryngology, GaziU niversity, Turkey

\section{Özgür Ekinci}

Department of Pathology, Gazi University, Turkey

\section{Osman Tugrul Güzeldir}

Department of Otorhinolaryngology, Gazi U niversity, Turkey

\section{Veysel Akif Savas}

Department of Otorhinolaryngology, Gazi U niversity, Turkey

\section{Ahmet Köybasioglu}

Professor, Department of Otorhinolaryngology, Gazi U niversity, Turkey 